\title{
OPEN Antagonism between brain regions relevant for cognitive control and emotional memory facilitates the generation of humorous ideas
}

\author{
Florian Bitsch ${ }^{1,2}$, Philipp Berger ${ }^{1,3}$, Andreas Fink $^{4}$, Arne Nagels ${ }^{1,5}$, Benjamin Straube ${ }^{1,2}$ \& \\ Irina Falkenberg ${ }^{1,2}$
}

The ability to generate humor gives rise to positive emotions and thus facilitate the successful resolution of adversity. Although there is consensus that inhibitory processes might be related to broaden the way of thinking, the neural underpinnings of these mechanisms are largely unknown. Here, we use functional Magnetic Resonance Imaging, a humorous alternative uses task and a stroop task, to investigate the brain mechanisms underlying the emergence of humorous ideas in 24 subjects. Neuroimaging results indicate that greater cognitive control abilities are associated with increased activation in the amygdala, the hippocampus and the superior and medial frontal gyrus during the generation of humorous ideas. Examining the neural mechanisms more closely shows that the hypoactivation of frontal brain regions is associated with an hyperactivation in the amygdala and vice versa. This antagonistic connectivity is concurrently linked with an increased number of humorous ideas and enhanced amygdala responses during the task. Our data therefore suggests that a neural antagonism previously related to the emergence and regulation of negative affective responses, is linked with the generation of emotionally positive ideas and may represent an important neural pathway supporting mental health.

Creative thinking can be considered a central building block of human achievements since it promotes the emergence of novel concepts by re-organizing established principles. One important aspect of creative thinking is the generation of humorous ideas ${ }^{1}$, which can be regarded as an emotionally positively laden component of divergent thinking ${ }^{2}$. Although the ability is deeply grounded in human social life, facilitating social relationships ${ }^{3}$ and promoting mental health ${ }^{4,5}$, its neural mechanisms are only beginning to be understood ${ }^{6}$. Studies examining the cognitive and neural mechanisms of divergent thinking have pointed out that cognitive control mechanisms can facilitate the mining of abstract ideas ${ }^{7}$. However, it is currently unclear, whether high ${ }^{8}$ or low inhibitory mechanisms ${ }^{9}$ enable the creative process ${ }^{2}$, and how these are represented in the brain. To shed light on the cognitive and neural mechanisms underlying the ability to generate humorous ideas, we use functional neuroimaging to examine humor generation and how it may be facilitated by inhibitory capacities.

In the context of divergent thinking, it has been suggested that the generation of creative ideas is closely interweaved with basic cognitive processes, such as cognitive control and executive functions ${ }^{7,10}$. More specifically, it has been assumed that during the generation of creative ideas, an active inhibition of content-related semantic mental representations facilitates a deeper search process to combine remote and therefore unusual knowledge structures within an associative semantic network ${ }^{11,12}$. This line of thinking received empirical support from studies showing that higher individual cognitive control capacities are associated with the formation of novel and abstract concepts ${ }^{13}$. Along these lines, some studies demonstrated that more creative individuals can be characterized by more flexible cognitive control, as indicated by greater trial-to-trial cognitive control modulation ${ }^{10,14}$. Furthermore, in a group of creative design students, an increased production of creative ideas was related with enhanced cognitive control abilities ${ }^{8}$. Interestingly, however, although broad consensus exists

\footnotetext{
${ }^{1}$ Department of Psychiatry and Psychotherapy, Philipps-University Marburg, Rudolf-Bultmann-Straße 8, 35039 Marburg, Germany. ${ }^{2}$ Center for Mind, Brain and Behavior - CMBB, Hans-Meerwein-Straße 6, 35032 Marburg, Germany. ${ }^{3}$ Department of Neuropsychology, Max Planck Institute for Human Cognitive and Brain Sciences, Stephanstraße 1a, 04103 Leipzig, Germany. ${ }^{4}$ Institute of Psychology, University of Graz, BioTechMed, Universitätsplatz 2, $8010 \mathrm{Graz}$, Austria. ${ }^{5}$ Department of English and Linguistics, Johannes Gutenberg-University Mainz, Jakob-Welder-Weg 18, 55128 Mainz, Germany. ${ }^{\varpi}$ email: bitschf@staff.uni-marburg.de
} 
that cognitive control processes are linked with the re-combination of established concepts to generate novel ideas, their specific contribution to the process of divergent thinking remains controversial ${ }^{2}$. Another line of research suggests that a reduction of cognitive control per se or a decoupling of inhibitory mechanisms is linked with a creative mind. This assumption was driven by findings of an overrepresentation of creative individuals in specific mental disorders in which dis-inhibition is a critical psychopathological facet, such as schizophrenia and bipolar disorders ${ }^{15-19}$. To simulate such a disinhibition effect, healthy subjects received a mild alcohol intoxication which led to a reduction in cognitive control capacities, but an increase in creative processes ${ }^{20}$. Along these lines, experimentally induced reduction of inhibitory cognitive control capabilities by exhaustive practice of related cognitive tasks, led to a higher originality and frequency of abstract ideas. While these findings suggest close links between idea generation processes and inhibitory mechanisms during the mining of abstract ideas, brain imaging techniques can provide additional insights into their interrelationship, thereby facilitating a more comprehensive understanding of the manifold neurocognitive mechanisms implicated in this complex mental ability domain.

During creative cognition, neuroimaging findings reveal activity patterns in a widespread neural network involving brain regions related with cognitive control mechanisms such as the superior frontal gyrus ${ }^{21}$, the inferior frontal gyrus $\mathrm{IFG}^{22,23}$, the dorsolateral prefrontal cortex $\mathrm{dlPFC}^{23,24}$ and brain regions associated with default mode processes such as the bilateral inferior parietal lobule (IPL), the posterior cingulate cortex (PCC), the medial prefrontal cortex (mPFC) and areas in the medial temporal lobe (MTL) such as the amygdala and the hippocampus, which are assumed to be critical for spontaneous and broadened thinking ${ }^{25-27}$. Humor production abilities have been found to have much in common with other forms of verbal creativity ${ }^{6,28}$. Accordingly, similar functional networks of brain regions are assumed in association with the production of humorous ideas (e.g. amygdala and hippocampus), and with humor comprehension processes (e.g. IFG, SFG and IPL ${ }^{29-31}$ ). Among these brain regions, the amygdala might have a central role for mining abstract concepts, given its implication for the acquisition, storage and modulation of emotional memory information ${ }^{32}$. Furthermore, the region is a central area in the processing of humor ${ }^{33,34}$. The degree to which inhibitory processes influence the regions' functions during the creative process ${ }^{23}$ is yet unknown. Previous research however suggests that the amygdala function is balanced by inhibition and disinhibition processes resulting in enhanced or reduced formation of emotions and cognition such as anxiety and fear ${ }^{32,35}$, a process particularly modulated by brain regions in the frontal cortex ${ }^{36}$.

Thus, a better understanding of whether inhibitory mechanisms modulate the mining of humorous ideas would shed light on the relationship between inhibitory mechanisms and memory-informed spontaneous $\operatorname{cognition}^{2}$ and extent our understanding of the mechanisms underlying humor generation. Here, we use a modified version of the alternative uses (AU) $\operatorname{task}^{37}$ as a well-established paradigm to assess the emergence of funny ideas. During the fMRI-task participants generated ideas for either funny or typical uses of everyday objects, while speech production was audio-recorded on-line. To examine whether inhibitory mechanisms modulate the mining of humorous ideas, we assessed each participant's cognitive control capacities with a color naming stroop task outside the MRI and examined their potential effect on the idea generation process. Previous humor research has shown the high relevance of the prefrontal cortex (PFC) in comprehension and appreciation of funny material $^{31,38}$. Besides the IFG ${ }^{31}$, the superior part of the frontal gyrus (SFG) has been associated with humour comprehension ${ }^{29,30}$. This region's specific function in humor comprehension is likely associated with its general relevance for higher-order cognitive processes, such as the organization of thoughts, schema-shifting and the development of inferences to form a new contex ${ }^{29}$. Additionally, the SFG has been found to be implicated in the appreciation of humor, indicating (i) that a complex cognitive process is linked with the appreciation phase ${ }^{39}$ and (ii) the region has a high relevance for humor in general. These findings suggest an important role of the SFG for the cognitive generation of humor, which we tested in our study.

We hypothesize that brain regions relevant for processing humor, such as the prefrontal cortex (particularly the SFG), the amygdala and the hippocampus, are implicated in the generation of funny (vs. typical) ideas.

This hypothesis will be tested by contrasting the functional activity during the generation of funny $>$ typical ideas. Furthermore, we expected that these brain regions and particularly prefrontal brain regions are modulated by inhibitory mechanisms which will facilitate the generation of humorous ideas. This hypothesis will be tested by investigating the effects of inhibitory abilities, as assessed by a stroop-task. Last, based on studies showing the high relevance of the medial and superior frontal ${ }^{29}$ and the amygdala ${ }^{25}$, we assumed that functional connectivity between the both regions will facilitate the flourishing of humorous ideas. Explorative analysis will examine whether there is an association between the neural underpinnings related to the production of funny ideas and personality differences in the regulation of emotional responses, given that an association between humorous abilities and emotion regulation can be expected ${ }^{4}$.

\section{Results}

Behavioral analysis. On average the participants generated $1.82 \pm 0.73(M \pm S T D)$ ideas per item in the funny condition and $2.27 \pm 0.79(M \pm$ STD) ideas per item in the typical condition. Participants produced more ideas in the typical condition than in the funny condition, $t(23)=2.52, p=0.02$.

fMRI analysis. Functional activity analysis: FUN $>$ TYP. The whole-brain analysis of funny versus typical idea generation (FUN $>$ TYP) revealed activation of a network spanning cortical and subcortical as well as cerebellar areas on both hemispheres, including the bilateral amygdala/hippocampus complex, left superior frontal and right medial gyrus, the bilateral anterior cingulate cortex (ACC), the bilateral inferior frontal gyrus (IFG) and the left temporal pole (Fig. 1a,b, Table 1). The reverse contrast (TYP $>$ FUN) revealed no significant suprathreshold clusters. 
a)

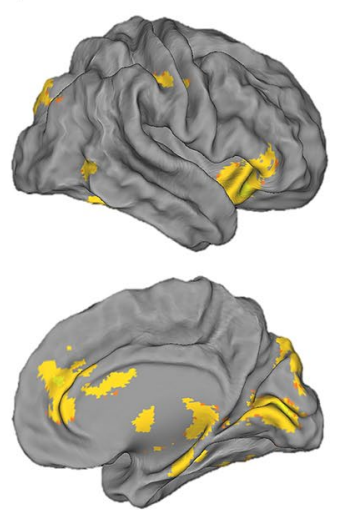

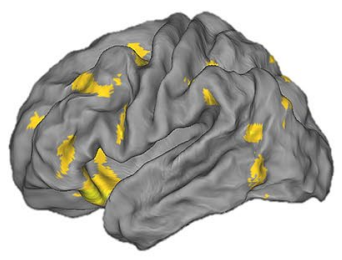

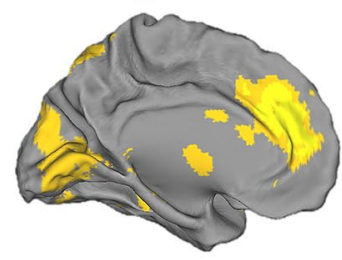

b)
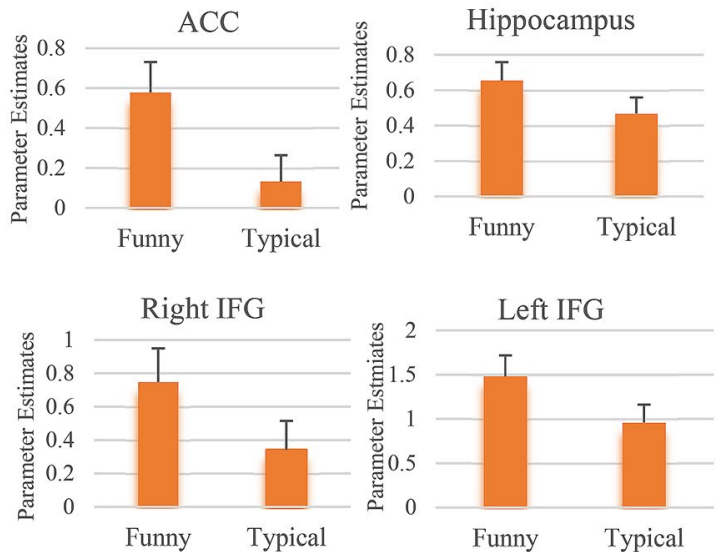

Figure 1. Functional activity analysis: (a) the functional activity analysis of the generating FUN $>$ TYP ideas contrast shows activity in a set of fronto-temporal brain regions ( $t$ values are plotted), such as $(\mathbf{b})$ presentation of the parameter estimates (mean beta values per condition) of the right ACC, the bilateral IFG and the left hippocampus. The error bars represent SEM.

Functional activity: covariate-analysis (stroop interference: FUN>TYP). Analysis of covariance revealed a positive relationship between enhanced cognitive control abilities (lower level of congruent-incongruent reaction times difference, Fig. 2b) and increased activity to humorous idea generation from the contrast FUN $>$ TYP in several clusters, including the right hippocampus and the right amygdala, the right superior/medial frontal gyrus, the left insula, the left superior temporal gyrus, the left IFG and the right putamen (see Fig. 2a,c, Table 2). There was no significant negative correlation between activity during the FUN > TYP condition and the stroop scores.

Functional connectivity: FUN > TYP. The functional connectivity analysis of the right superior/medial frontal gyrus showed a significant association with the left middle frontal gyrus, the right nucleus caudate and the left posterior insula (Table 3).

Functional connectivity: covariate-analysis (stroop interference: FUN $>$ TYP). In a next step, the impact of cognitive control abilities on functional connectivity of the right superior/medial frontal gyrus was assessed, by examining the effects of interindividual differences in cognitive inhibition as covariate of interest. This analysis revealed a reduced functional connectivity between the right superior/medial frontal gyrus and the left amygdala (small volume corrected, $t=4.46, p_{F W E}=0.009$, Fig. 3 ) and the left postcentral and bilateral precentral gyrus in participants with enhanced cognitive control abilities (Table 4).

Amygdala activity: superior/medial frontal gyrus connectivity correlations. To investigate a potential increase of the amygdala activity by a negative functional coupling with prefrontal brain regions, we performed a correlation analysis between functional activity of the left amygdala and its functional connectivity with the right superior/medial frontal gyrus. This analysis revealed a significant negative relationship between the left amygdala activity and its connectivity with the right superior and right medial frontal gyrus $(r=-0.57, p<0.05$, Fig. $4 \mathrm{~d})$ indicating a stronger functional activity increase of the left amygdala by a higher negative frontal coupling. The general relevance of this amygdala region for the generation of humorous ideas is shown by its enhanced activity in the funny compared to the typical condition (Region-of-Interest Analysis, $t=1.73, p=0.048$, Fig. 4a).

Brain-behavior and traits correlations. Correlations of extracted beta estimates showed a significant association between the left amygdala activity with the number of generated ideas in the funny condition $(r=0.50, p<0.05$, Fig. 4b). Furthermore, the quantity of participants' produced ideas during the funny condition correlated negatively with functional connectivity measures of the amygdala-superior/medial frontal gyrus $(r=-0.44, p<0.05$, Fig. 4c), pointing to an increased productivity by a reduced functional connectivity. Additionally, we examined a potential association between the activity of the amygdala and behavioral tendencies to regulate emotions in real-life. This analysis showed that a suppressive emotion-regulation tendency $(\mathrm{ERQ})(\mathrm{r}=-0.49, \mathrm{p}<0.05)$ is associated with reduced left amygdala activity during the production of funny ideas.

\section{Discussion}

In employing a novel humorous idea generation task and a classic stroop task, we provide first evidence for the neural mechanisms underlying the production of funny ideas. Our results reveal that humorous idea production is associated with a brain circuit involving the bilateral amygdala/hippocampus, the right superior/medial frontal gyrus, the left insula and the bilateral IFG. Remarkably, enhanced cognitive control abilities were associated with increased neural responses in the similar brain regions during the humor generation process, pointing to a facilitative effect of cognitive control abilities and abstract thinking. More specifically, we were able to demonstrate that 


\begin{tabular}{|c|c|c|c|c|c|c|c|}
\hline \multirow[b]{2}{*}{ Region } & \multirow[b]{2}{*}{ Anatomical region } & \multirow[b]{2}{*}{ Hemisphere } & \multirow[b]{2}{*}{ Voxel per cluster } & \multicolumn{3}{|c|}{ Peak coordinates } & \multirow{2}{*}{\begin{tabular}{|l} 
T-value \\
$\mathrm{T}$ \\
\end{tabular}} \\
\hline & & & & $\mathrm{x}$ & $\mathbf{y}$ & $\mathbf{z}$ & \\
\hline \multirow{3}{*}{ Cluster 1} & Hippocampus & Right & 7462 & 38 & -24 & -8 & 5.61 \\
\hline & Parahippocampal gyrus & Right & & 24 & -24 & -18 & 5.60 \\
\hline & Cerebellum & Right & & 36 & -54 & -30 & 5.38 \\
\hline \multirow{7}{*}{ Cluster 2} & Calcarine gyrus & Right & 6578 & 18 & -78 & 6 & 7.83 \\
\hline & Middle temporal gyrus & Left & & -42 & -56 & 10 & 5.37 \\
\hline & Cerebellum & Left & & -28 & -76 & -36 & 5.05 \\
\hline & Calcarine gyrus & Left & & -10 & -84 & 10 & 4.94 \\
\hline & Precuneus & Left & & -6 & -76 & 52 & 4.82 \\
\hline & Cuneus & Left & & -4 & -90 & 32 & 4.78 \\
\hline & Middle occipital gyrus & Left & & -38 & 68 & 22 & 4.74 \\
\hline \multirow{4}{*}{ Cluster 3} & ACC & Right & 4634 & 10 & 46 & 27 & 6.76 \\
\hline & Superior medial gyrus & Right & & -8 & 48 & 18 & 6.26 \\
\hline & ACC & Left & & -10 & 32 & 28 & 5.91 \\
\hline & \begin{tabular}{|l|} 
Superior frontal gyrus \\
\end{tabular} & Left & & -18 & 58 & 18 & 5.33 \\
\hline \multirow{3}{*}{ Cluster 4} & IFG & Left & 1617 & -32 & 32 & -16 & 6.06 \\
\hline & Insula & Left & & -36 & 16 & -14 & 5.69 \\
\hline & \begin{tabular}{|l|} 
Temporal pole \\
\end{tabular} & Left & & -48 & 12 & -6 & 3.96 \\
\hline Cluster 5 & IFG & Right & 1372 & 38 & 28 & -10 & 7.09 \\
\hline \multirow{2}{*}{ Cluster 6} & Cerebellum & Left & 379 & -10 & -56 & -36 & 4.52 \\
\hline & Cerebellum & Right & & 10 & -50 & -42 & 4.49 \\
\hline \multirow{2}{*}{ Cluster 7} & Inferior parietal lobule & Left & 372 & -54 & -38 & 36 & 4.59 \\
\hline & Postcentral gyrus & Left & & -52 & -30 & 58 & 4.43 \\
\hline \multirow{2}{*}{ Cluster 8} & Postcentral gyrus & Right & 280 & 62 & -18 & 46 & 4.65 \\
\hline & Precentral gyrus & Right & & 48 & -16 & 44 & 3.98 \\
\hline Cluster 9 & Inferior frontal gyrus & Left & 145 & -46 & 10 & 14 & 5.30 \\
\hline Cluster 10 & Hippocampus & Left & 81 & -36 & -28 & -8 & 4.30 \\
\hline
\end{tabular}

Table 1. Clusterwise statistics of the Contrast FUN > TYP. All reported regions were corrected for multiple comparisons on a cluster threshold of $\mathrm{p}<0.05$, voxel threshold $\mathrm{p}=0.001, \mathrm{k}=47$ voxel. Clusters which survived a FDR-correction are indicated by $\mathrm{a}+$. Coordinates are reported in MNI space.

a)
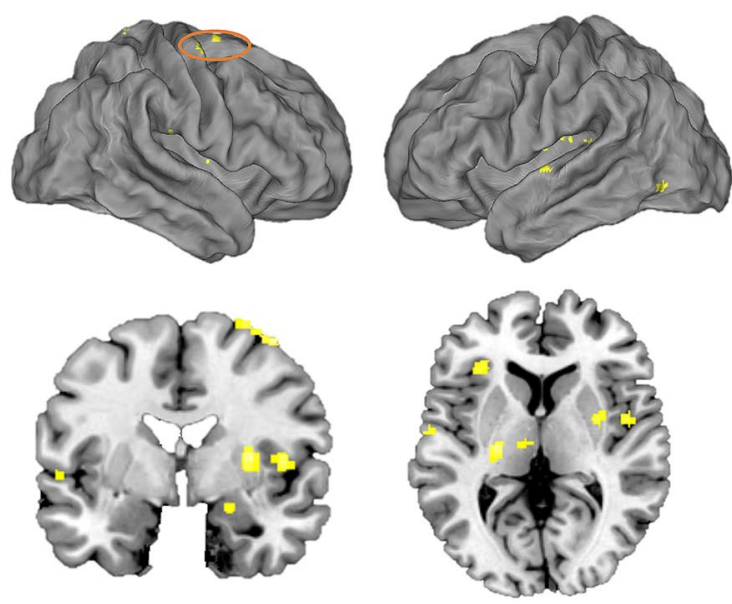

b)

c)
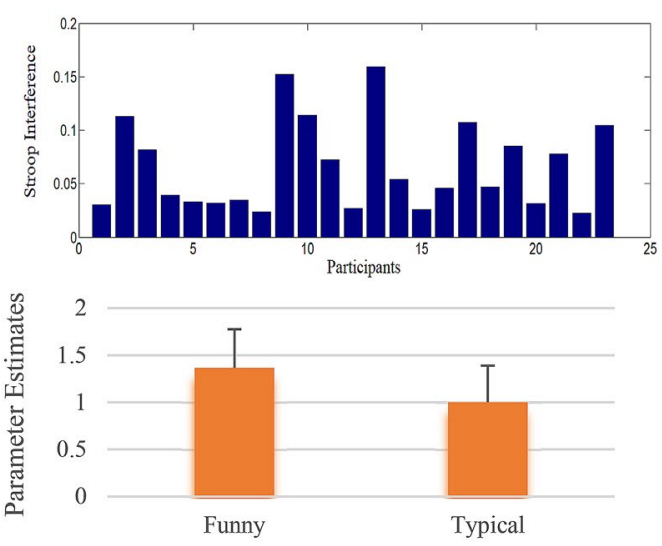

Figure 2. Functional activity: covariate analysis (stroop interference): (a) the functional covariation analysis of the stroop interference in the FUN > TYP contrast revealed a positive relationship between individual differences in cognitive inhibition and activation in several brain regions associated with humorous processing ( $t$ values are plotted). (b) Distribution of the stroop interference values per participant. The value represents the difference between reaction times in the congruent vs. incongruent word-color pairs condition. (c) Functional activity of the superior/medial frontal gyrus, the cluster was used for the subsequent connectivity analysis. Error bars represent SEM. 


\begin{tabular}{|c|c|c|c|c|c|c|c|}
\hline \multirow{2}{*}{\multicolumn{2}{|c|}{\begin{tabular}{l|l} 
Region & Anatomical region \\
Reduced stroop interference
\end{tabular}}} & \multirow[b]{2}{*}{ Hemisphere } & \multirow[b]{2}{*}{ Voxel per cluster } & \multicolumn{3}{|c|}{ Peak coordinates } & \multirow{2}{*}{\begin{tabular}{|l|} 
T-value \\
T
\end{tabular}} \\
\hline & & & & $\mathrm{x}$ & $\mathbf{y}$ & $\mathbf{z}$ & \\
\hline Cluster 1 & Cerebellum & Left & 539 & -12 & -50 & -20 & 4.56 \\
\hline Cluster 2 & Postcentral gyrus & Right & 102 & 18 & -36 & 80 & 4.77 \\
\hline \multirow{2}{*}{ Cluster 3} & Superior frontal gyrus & Right & 101 & 26 & 2 & 70 & 4.85 \\
\hline & Middle frontal gyrus & Right & & 40 & 0 & 64 & 4.42 \\
\hline \multirow{2}{*}{ Cluster 4} & Hippocampus & Right & 97 & 20 & -6 & -18 & 4.73 \\
\hline & Amygdala & Right & & 26 & -6 & -10 & 4.31 \\
\hline Cluster 5 & Putamen & Right & 92 & 32 & -4 & 10 & 4.56 \\
\hline \multirow{2}{*}{ Cluster 6} & Thalamus & Right & 91 & 2 & -14 & 0 & 4.05 \\
\hline & Thalamus & Left & & -10 & -18 & 2 & 3.88 \\
\hline Cluster 7 & Rolandic operculum & Right & 75 & 46 & -2 & 6 & 4.26 \\
\hline Cluster 8 & Insula & Left & 72 & -32 & -10 & 14 & 3.98 \\
\hline Cluster 9 & Superior temporal gyrus & Left & 65 & -58 & -10 & 2 & 3.98 \\
\hline Cluster 10 & Supramarginal gyrus & Right & 64 & 52 & -20 & 24 & 4.33 \\
\hline Cluster 11 & Inferior occipital gyrus & Left & 57 & -48 & -70 & -6 & 3.87 \\
\hline Cluster 12 & Inferior frontal gyrus & Left & 48 & -32 & 30 & 6 & 4.24 \\
\hline
\end{tabular}

Table 2. Clusterwise statistics of the analysis of covariance of the individual stroop score with the FUN $>$ TYP contrast. All reported regions were corrected for multiple comparisons on a cluster threshold of $\mathrm{p}<0.05$, voxel threshold $\mathrm{p}=0.001, \mathrm{k}=47$ voxel. Coordinates are reported in MNI space.

\begin{tabular}{|c|c|c|c|c|c|c|c|}
\hline \multirow[b]{2}{*}{ Region } & \multirow[b]{2}{*}{ Anatomical region } & \multirow[b]{2}{*}{ Hemisphere } & \multirow{2}{*}{$\begin{array}{l}\text { Voxel per cluster } \\
\mathbf{k}\end{array}$} & \multicolumn{3}{|c|}{ Peak coordinates } & \multirow{2}{*}{\begin{tabular}{|l} 
T-value \\
T \\
\end{tabular}} \\
\hline & & & & \begin{tabular}{|l|}
$x$ \\
\end{tabular} & $\mathbf{y}$ & $\mathbf{z}$ & \\
\hline Cluster 1 & Middle frontal gyrus & Left & 96 & -26 & 18 & 38 & 4.32 \\
\hline Cluster 2 & Nucleus caudate & Right & 85 & 16 & 4 & 16 & 4.59 \\
\hline Cluster 3 & Insula (posterior) & Left & 62 & 34 & -34 & 11 & 4.51 \\
\hline
\end{tabular}

Table 3. Clusterwise statistics of the functional connectivity analysis with the FUN $>$ TYP contrast. All reported regions were corrected for multiple comparisons on a cluster threshold of $\mathrm{p}<0.05$, voxel threshold $\mathrm{p}=0.001, \mathrm{k}=47$ voxel. Coordinates are reported in MNI space.

participants with enhanced cognitive control abilities also displayed stronger negative functional connectivity between brain regions related to inhibitory processes (e.g. right the superior/medial frontal gyrus ${ }^{21}$ ) and the left amygdala, a key region for humor processing ${ }^{25,31}$. Notably, the stronger the negative connectivity between the amygdala and brain regions related to cognitive control (i.e. right superior/medial frontal gyrus) was, the greater the activity in the left amygdala increased during funny idea generation. This finding is suggestive of an antagonism between brain regions related to cognitive control and the amygdala, leading to an increased availability of funny ideas. This interpretation is motivated by the finding that amygdala activity was linked both with outcome measures of the idea generation process, since participants with higher amygdala responses produced a higher number of funny ideas and a tendency to use suppressive emotion-regulation strategies as a coping mechanism in real-life. Notably, our findings suggest that the well-established association between neural pathways linking cognitive control and affective systems ${ }^{40}$, which has been found to be associated with increased negative emotional responses ${ }^{36}$, is also relevant for the production of positive affective cognition and may therefore be particularly relevant for mental health ${ }^{41}$. Recent research about emotional evaluation shows that the left amygdala is linked with finding novel relationships which are accompanied by positive emotional valence $^{42}$. In this sense the building of novel positive associations, might reflect the amygdala's relevance in our findings. Studies examining the cognitive and neural mechanisms of divergent thinking show that cognitive control mechanisms can facilitate the mining of abstract ideas ${ }^{7}$. However, it has been speculated whether high ${ }^{8}$ or low inhibitory mechanisms $s^{9}$ enable the creative process ${ }^{2}$ and how these are represented in the brain. The assumption of a positive association between cognitive control processes and enhanced creativity ${ }^{13,43}$ was supported by empirical findings showing that individuals with elaborated creative abilities also possess high cognitive control abilities in a stroop task ${ }^{8}$. Moreover, a positive association between fluid intelligence and divergent thinking has been reported and linked to a higher creative potential ${ }^{13}$. The findings led to the theoretical interpretation that creative individuals are better able to suppress competing and obvious semantic associations, which is assumed to increase the availability of abstract concepts in the consciousness stream ${ }^{8}$.

Adding to this picture, our imaging data indicate that participants with enhanced cognitive control abilities showed an increased left amygdala activity but a negative connectivity with the right superior frontal/ medial gyrus, while generating humorous ideas. This finding suggests that an activity increase in the amygdala 
a)
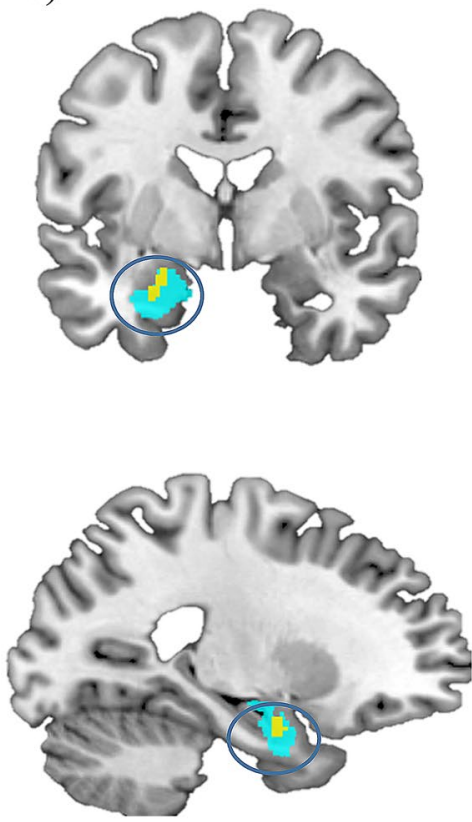

b)

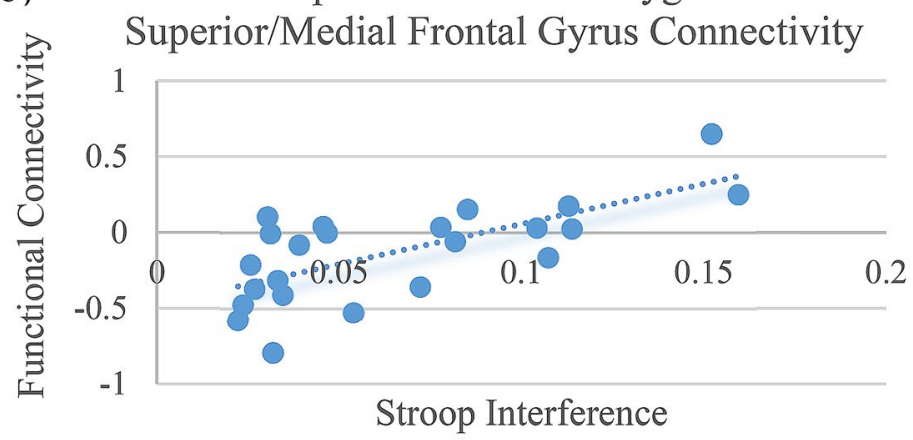

c)

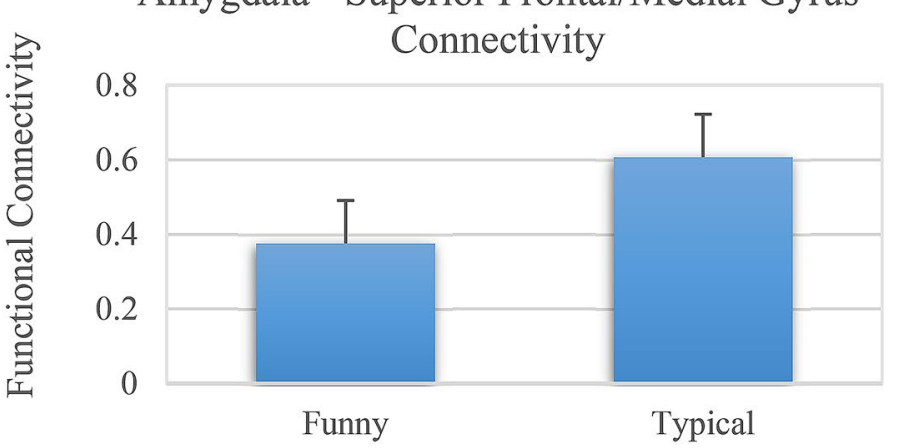

Figure 3. Functional connectivity: covariate analysis (stroop interference): (a) significant functional connectivity between the right superior frontal/medial gyrus and the left amygdala (turquoise anatomically defined region-of-interest, yellow significantly activated amygdala (SMV-corrected)). (b,c) Parameter Estimates were extracted from the significant functional amygdala cluster, the error bars represent SEM.

\begin{tabular}{|c|c|c|c|c|c|c|c|}
\hline Region & Anatomical region & \multirow[b]{2}{*}{ Hemisphere } & \multirow{2}{*}{\begin{tabular}{|l|} 
Voxel per cluster \\
$\mathbf{k}$
\end{tabular}} & \multicolumn{3}{|c|}{ Peak coordinates } & \multirow{2}{*}{\begin{tabular}{|l|} 
T-value \\
$\mathrm{T}$ \\
\end{tabular}} \\
\hline \multicolumn{2}{|c|}{ Increased stroop interference } & & & $\mathbf{x}$ & $\mathbf{y}$ & $\mathbf{z}$ & \\
\hline \multirow{2}{*}{ Cluster $1^{+}$} & Precentral gyrus & Right & 373 & 20 & -20 & 70 & 6.33 \\
\hline & Superior frontal gyrus & Right & & 32 & -12 & 62 & 4.02 \\
\hline \multirow{2}{*}{ Cluster $2^{+}$} & Precentral gyrus & Left & 371 & -48 & -2 & 34 & 6.03 \\
\hline & Postcentral gyrus & Left & & -46 & -18 & 30 & 4.32 \\
\hline Small-volume correction & Amygdala & Left & 20 & -24 & -2 & -24 & 4.46 \\
\hline
\end{tabular}

Table 4. Clusterwise statistics of the functional connectivity analysis of covariance of the individual stroop score (reduced stroop interference) with the FUN $>$ TYP contrast. All reported regions were corrected for multiple comparisons on a cluster threshold of $\mathrm{p}<0.05$, voxel threshold $\mathrm{p}=0.001, \mathrm{k}=47$ voxel. Clusters which survived a FDR-correction are indicated by $\mathrm{a}+$. Coordinates are reported in MNI space.

is associated with a superior frontal/medial gyrus activity decrease, and vice versa. This antagonistic connectivity was linked with a higher activity in the amygdala and related to an increase in idea generation. Our results might therefore facilitate further research in psychiatric patients particularly schizophrenia, bipolar disorder or ADHD. This line of research has shown that if cognitive and behavioral disinhibition is a core characteristic of the disorder, there is also a tendency for enhanced creative potential ${ }^{16-18}$. A unifying element of disorders such as bipolar disorders $^{44,45}, \mathrm{ADHD}^{46,47}$ and schizophrenia ${ }^{48,49}$ are functional and structural alterations in frontal brain regions associated with cognitive control processes. Taking these findings together, the studies suggest that an imbalance in inhibitory and disinhibitory processes is linked with a hypersensitivity in limbic structures ${ }^{50,51}$, which in turn is assumed to be associated with higher creativity ${ }^{16,17,23}$. Interestingly, our data also suggests that a stronger amygdala response during the generation of emotionally positive ideas is associated with an increased real-life usage of positive emotion-regulation strategies. The association with a reduced suppression of positive emotional responses has been shown to be related to beneficial mental health outcomes ${ }^{4}$. Hence, other emotion-regulation strategies might be preferred by these individuals or they have stronger experience of positive emotions, which might be related to the generation and experience of humorous ideas. This association should therefore be further examined in psychiatric patients as, for example, interventions promoting humorous coping ${ }^{5}$ may to facilitate positive emotions in this group. 
a)

a

c)

Generated Ideas - Amygdala-

Superior/Medial Frontal Gyrus

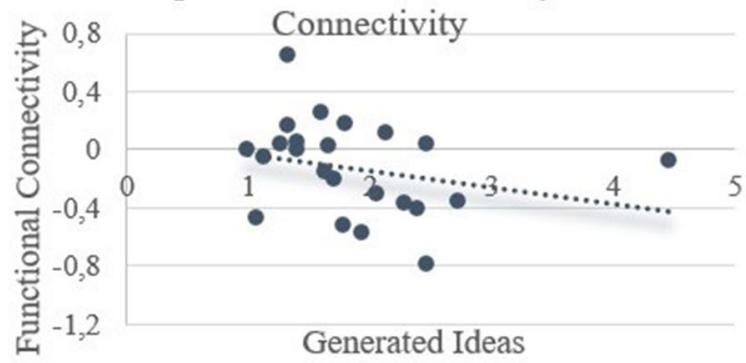

b) Generated Ideas - Amygdala Activity

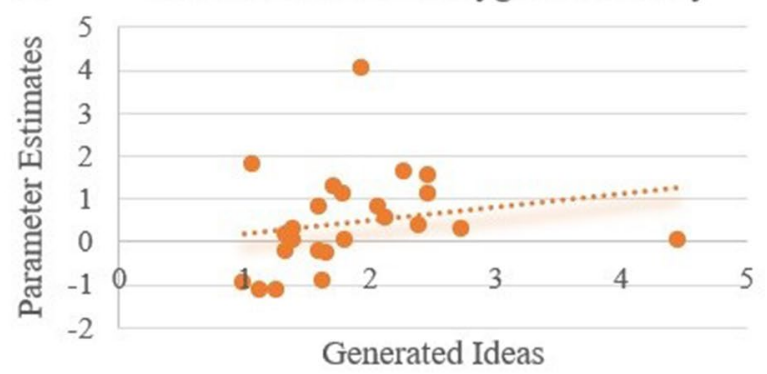

Amygdala Activity and AmygdalaSuperior/Medial Frontal Gyrus Connectivity

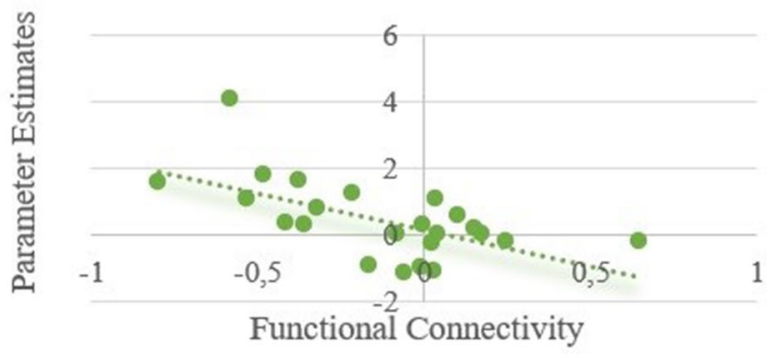

Figure 4. (a) Significant higher functional activity in the amygdala in the funny vs. typical condition, the error bars represent SEM. (b) Higher amygdala activity was associated with increased generation of funny idea. (c) Reduced functional connectivity between the amygdala and the superior/medial frontal gyrus was associated with increased funny idea generation (d) A higher negative functional connectivity between the amygdalasuperior/medial frontal gyrus was associated with an increased functional activity in the amygdala.

From a conceptual perspective, it has been suggested that frontal brain regions might facilitate the re-structuring of memory processes related to creative cognition ${ }^{2}$. This notion is somewhat contradictory to our finding of reduced frontal-amygdala connectivity being associated with an increased availability of funny ideas. Previous studies have pointed to a strong anatomical interconnectivity between the amygdala and the frontal cortex ${ }^{52}$, suggesting that frontal brain regions are implicated in the functional regulation of negative emotional and cognitive experiences, such as anxiety, triggered by the amygdala ${ }^{53,54}$. Accordingly, our data indicate that in the domain of generating positive ideas a similar frontal hypoactivity is associated with an amygdala hyperactivity, and vice versa, a neural antagonism which leads to an increase in the availability of positive ideas. Our interpretation is strengthened by an increased positive coupling of the amygdala with frontal brain regions during the control condition in which familiar and non-emotional concepts had to be retrieved. This suggests that in general inhibitory mechanisms of the amygdala control the expression of emotional associations ${ }^{55,56}$. Given that the amygdala is reliably found in humor processing ${ }^{31}$ and considered a target of inhibitory mechanisms to regulate emotional states $^{32}$ and memory processes ${ }^{57}$, we suggest that the regions key relevance during the generation of humor evolves by a dynamic coupling with brain regions relevant for inhibitory processes. Along this line, our findings open-up the question whether similar mechanisms can be found in clinical populations with schizophrenia, bipolar disorder or $\mathrm{ADHD}^{18}$.

Some limitations have to be considered by interpreting the findings of this study. First, this fMRI-study with 24 participants had a relatively small sample size $e^{58,59}$. However, from a statistical point of view a small sample size might be less problematic to detect psychologically important effects ${ }^{60}$. However, a replication and extension of the findings in other populations is desirable. Additionally, there is a broad range of contexts where humorous and creative skills can evolve. The task used in this study is one of them. Hence, it is straightforward to extend the implications of our findings to different experimental tasks and ecological settings ${ }^{28}$. In order to improve the ecological validity of the task, future studies might include a funniness rating in the AU task, to link the self-experienced funniness to the generated ideas. This would extend the insight of brain process underlying the production of funny material. This might be also facilitated by improving fMRI settings and accessories (e.g. more channels for the head-coil).

With this study we provide the neurobiological key regions for the examination of related processes in clinical populations which might be extended by collecting the participants' immediate experienced emotional state after generating humorous ideas in future studies.

\section{Materials and methods}

Participants. 24 participants ( 12 females) between 23 and 56 years of age $(M \pm S T D=32.71 \pm 9.66)$ participated in the study. The sample was recruited via advertisements and e-mail distributors from (and to represent) the general population. All participants had normal or corrected-to-normal vision, reported no history of neurological or psychiatric disorders (which was excluded by a structured clinical interview for DSM-IV) and 


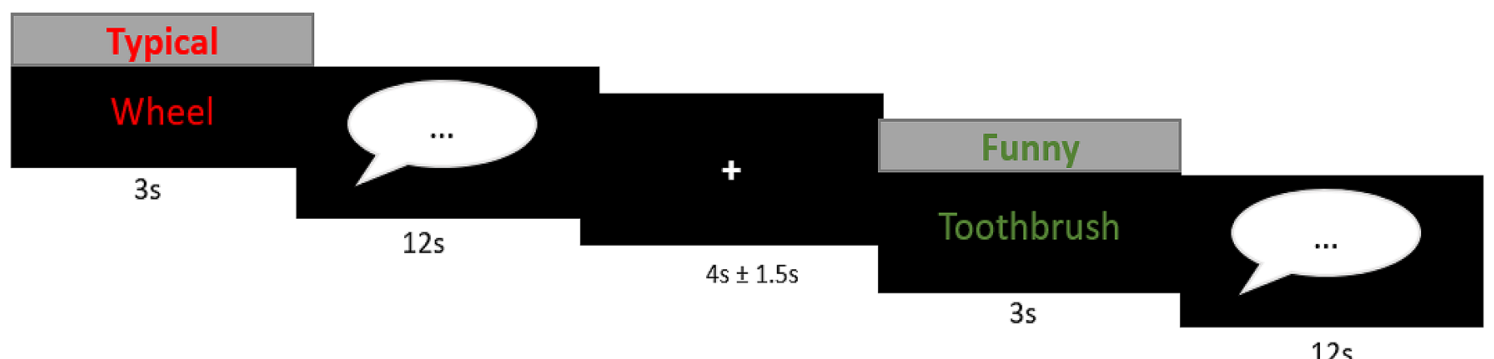

Figure 5. Experimental paradigm. During the task participants had to find either funny or typical usages for everyday objects ( 15 objects per condition). First participants were presented with the condition and word, afterwards they had to think aloud their idea. The ideas were recorded with a fMRI-suitable microphone and on-line transcripted.

were right-handed according to Edinburgh Handedness Inventory ${ }^{61}$. The study was approved by the local Ethics Committee at Philipps-University Marburg. All experiments were performed in accordance with the relevant guidelines and the Declaration of Helsinki. All participants gave written informed consent prior to the experiment and were reimbursed for the time they spent at the study site.

Experimental task. Participants performed an adapted alternative uses (AU) task, where they were presented with 30 names of everyday objects written in either green (funny (FUN) condition, 15 items) or red (typical (TYP) condition, 15 items) font color for the duration of $3 \mathrm{~s}$, followed by a speech bubble for the duration of $12 \mathrm{~s}$ (see Fig. 5). Participants were instructed to generate a high quantity and quality of humorous ideas or typical uses of the presented items, depending on the condition, as soon as the speech bubble occurred. The participants verbally reported the ideas that spontaneously came to their mind e. g. balloon: "I put it under my shirt that it seems that I am overweighted or pregnant" (FUN condition). Or in the TYP condition: e.g. soap: "I use it to wash myself". This procedure allows the identification of brain processes related to the generation of funny and creative ideas vs. recalling the typical usage of everyday objects. After the participants have reported their ideas, a fixation cross was presented (jittered for $4 \pm 1.5 \mathrm{~s}$ ) where participants could relax and subsequently process and respond to the other condition. In total, two different word lists were used in the task, which were pseudorandomly assigned to the participants. Given that no rating of the experienced funniness during the generation of ideas was collected, the task allows mainly to examine brain process related to the generation of funny and creative ideas.

Verbal answers were audio-taped during the scanning procedure and verbal output during the speech phase was transcripted using FOLKER ${ }^{62}$. Afterwards, the amount of (i) generated words and (ii) ideas (fluency score) was assessed. For each item, the amount of generated ideas was rated as follows: e.g. for the item "picture frame" a participant responded: „Carving the picture-frame by oneself, put oneself into the picture frame”. This answer was counted as two produced ideas for this participant and item.

Individual cognitive control abilities. To assess the participant's ability to inhibit cognitive interference of impeding stimuli-features, we applied the Stroop Color and Word Test, outside and following the MRI. The task was programmed and run within the Psychtoolbox implemented in Matlab (http://psychtoolbox.org/). The task included 4 words and colors: blue, green, red, yellow and the participants had to respond within $1.3 \mathrm{~s}$ to the color displayed (with one of four keys). The stroop interference score ${ }^{63}$, which is the ratio between the correct reaction times in the congruent condition (word and color identical) vs. the incongruent condition (word and color differently), was subsequently calculated and used a as an indicator for each participant's inhibitory performance.

Individual differences in emotion regulation and coping. To examine the participants' differences in regulating emotional experiences and stressful life-events, we assessed the emotion regulation questionnaire $\left(E^{6}{ }^{64}\right)$ following the fMRI-session. The ERQ is a 10-items self-report which measures two behavioral tendencies how individuals make use of two different emotion regulation process either reappraisal (6-items) or suppression (4-items).

Imaging procedure. MRI data acquisition. All images were acquired using a Siemens 3-T Trio with a 12-channel head matrix receive coil. Functional images were acquired using a $\mathrm{T}^{\star}{ }^{\star}$ weighted single shot echo planar imaging (EPI) sequence (parallel imaging factor of 2 (GRAPPA), TE $=30 \mathrm{~ms}, \mathrm{TR}=2000 \mathrm{~ms}$, flip angle $90^{\circ}$, slice thickness $3.6 \mathrm{~mm}$, matrix $64 \times 64$, in-plane resolution $3 \times 3 \mathrm{~mm}^{2}$, bandwidth $2232 \mathrm{~Hz} /$ pixel, EPI factor of 64 and an echo spacing of $0.51 \mathrm{~ms}$ ). Data from 33 transversal slices oriented to the AC-PC line were gathered in descending order. Additionally, a whole head T1 weighted data set was acquired with a 3D MP-Rage sequence (parallel imaging factor of 2 (GRAPPA), TE $=2.26 \mathrm{~ms}$, TR $=1900 \mathrm{~ms}$, flip angle $9^{\circ}, 1 \mathrm{~mm}$ isometric resolution, 176 sagittal slices, $256 \times 256$ matrix). In total 295 volumes were acquired. 
fMRI data preprocessing. Functional data preprocessing and analysis were performed using SPM12 (http:// www.fil.ion.ucl.ac.uk/spm) implemented in MATLAB 13a (MathWorks, MA). The first five volumes of each functional run were discarded from the analysis to account for T1 equilibration effects. Functional data were realigned and unwarped, corrected for slice timing, spatially normalized onto a common brain space (Montreal Neurological Institute, MNI) and spatially smoothed using a Gaussian filter with an $8 \mathrm{~mm}$ full-width half maximum (FWHM) Gaussian kernel. In order to improve fMRI-data quality, an automated quality assurance protocol (Artifact (ART) Detection toolbox, https://www.nitrc.org/projects/artifact_detect/), based on head motion $(>2 \mathrm{~mm}$ ) or aberrant signal intensity (global-signal z-value exceeded a threshold of 9) was used. Participants had on average $8.95 \pm 21.47$ affected scans. The ART-parameters were subsequently included in the first-level fMRI analysis. No participant had more than $33 \%$ affected scans, which is why all participants were included in the analysis.

fMRI data analysis. For statistical analysis we set up a general linear model (GLM) in SPM12 on the subject level, with 4 regressors comprising the onsets of the four task events (word: funny and typical $(3 \mathrm{~s})$, speech: funny and typical (12 s), Fig. 5). The number of words spoken were included as parametric modulator in the speech condition to control for speech related activity. Subsequently, the data were high-pass filtered with a cut-off frequency of $128 \mathrm{~s}$. To control for movement related activity, the realignment parameters and art parameters (see "fMRI data preprocessing" section in "Materials and methods" section) were included in the first-level model. Contrasts of interests were the funny (FUN) > typical (TYP) condition (speech and word phase), which were calculated during the first-level analysis. Afterwards the related contrast images were included in a second level model (one sample t-test). To correct for multiple voxel comparisons an extent threshold was used via Monte-Carlo simulation ${ }^{65}$. The whole-brain activation was simulated assuming a type I error voxel activation of $\mathrm{p}=0.001$, this revealed a cluster extent of 47 contiguous resampled voxels as sufficient to correct for multiple comparisons at $\mathrm{p}<0.05$. Additionally, the contrasts were corrected for multiple comparisons with a FDR method, this is specified in the tables.

Functional analysis of covariance. To investigate the relationship between inhibitory skills and the creative idea generation process during the FUN vs. TYP condition, we additionally conducted correlation analyses by including the individual stroop scores (see the "Individual cognitive control abilities" section in "Materials and methods" section) as a covariate of interest in a separate second level GLM. One participant has not conducted the stroop task and was therefore excluded from the covariate analysis. The correction for multiple comparisons in this analysis where the same as in the functional activation analysis (please see "fMRI data preprocessing" section in "Materials and methods" section).

Functional connectivity. We assessed task-related connectivity changes with the generalized form of contextdependent psychophysiological interaction $(\mathrm{gPPI})^{66}$. The gPPI approach is assumed to have a higher sensitivity and specificity due to a better model fit compared to the standard form of PPI analysis ${ }^{66}$. For the connectivity analysis, a functionally defined region of interest (ROI) of the right superior/medial frontal gyrus from the analysis of covariance (see "Functional activity: covariate analysis (stroop interference: FUN>TYP)" section in "Results" section) was used as seed region. The region's eigenvariate was extracted and for each condition a PPIregressor was built and included in a first-level model. This first-level model consisted of the regressors from the functional activity analysis (described above) in addition to the seed-region's time course. Contrast maps for FUN > TYP were calculated subsequently for each participant and submitted to a second-level random effects analysis (one sample $t$-test) including the stroop score as a covariate of interest (see "Functional activity: covariate analysis (stroop interference: FUN>TYP)" section in "Results" section), analogous to the functional activity analysis. The correction for multiple comparisons where in this analysis the same as in the functional activation analysis (please see in "fMRI data preprocessing" section).

Correlation analysis. In order to examine a potential relationship between functional activity and functional connectivity measures with the idea generation processes (quantity of funny ideas), we extracted the beta estimates from the entire functionally defined cluster of the amygdala and the superior/medial frontal gyrus and conducted subsequent correlation analysis by skipped correlations ${ }^{67,68}$ using the Matlab based robust correlation toolbox (http://sourceforge.net/projects/robustcorrtool/), which detects and disregards outliers depending on the data structure ${ }^{67}$. An additional explorative correlation analysis between the amygdala activity and amygdalaSFG/MFG allows to test whether intra-individual association between the functional activity with the connectivity (modulated by cognitive control) exists. Based on the intra-individual covariance we might show that in the same participants a higher amygdala activity is associated with a lower amygdala-SFG/MFG functional connectivity, which would provide an important insight into the findings. All correlation analyses were corrected for multiple comparisons with a Bonferroni correction. This analysis showed that all analyses remain significant after correcting for the 4 conducted tests (Supplementary Material).

Received: 8 August 2020; Accepted: 28 April 2021

Published online: 21 May 2021 


\section{References}

1. Ruch, W. \& Heintz, S. Humor production and creativity: Overview and recommendations. In Creativity and Humor (eds Ruch, W. \& Heintz, S.) 1-42 (Elsevier, Berlin, 2019).

2. Benedek, M. \& Fink, A. Toward a neurocognitive framework of creative cognition: The role of memory, attention, and cognitive control. Curr. Opin. Behav. Sci. 27, 116-122 (2019).

3. Berger, P., Bitsch, F., Bröhl, H. \& Falkenberg, I. Play and playfulness in psychiatry: A selective review. Int. J. Play 7(2), 210-225. https://doi.org/10.1080/21594937.2017.1383341 (2018).

4. Samson, A. C., Glassco, A. L., Lee, I. A. \& Gross, J. J. Humorous coping and serious reappraisal: Short-term and longer-term effects. Eur. J. Psychol. 10(3), 571-581 (2014).

5. Falkenberg, I., McGhee, P. E. \& Wild, B. Humorfähigkeiten trainieren: Manual für die psychiatrisch-psychotherapeutische Praxis (Schattauer, 2013).

6. Perchtold-Stefan, C. M. et al. Humor comprehension and creative cognition: Shared and distinct neurocognitive mechanisms as indicated by EEG alpha activity. Neuroimage 213, 116695. https://doi.org/10.1016/j.neuroimage.2020.116695 (2020).

7. Jung, R. E., Mead, B. S., Carrasco, J. \& Flores, R. A. The structure of creative cognition in the human brain. Front. Hum. Neurosci. 7, 330. https://doi.org/10.3389/fnhum.2013.00330 (2013).

8. Edl, S., Benedek, M., Papousek, I., Weiss, E. M. \& Fink, A. Creativity and the stroop interference effect. Person. Individ. Differ. 69, 38-42. https://doi.org/10.1016/j.paid.2014.05.009 (2014).

9. Radel, R., Davranche, K., Fournier, M. \& Dietrich, A. The role of (dis)inhibition in creativity: Decreased inhibition improves idea generation. Cognition 134, 110-120. https://doi.org/10.1016/j.cognition.2014.09.001 (2015).

10. Zabelina, D. L. \& Robinson, M. D. Creativity as flexible cognitive control. Psychol. Aesthet. Creat. Arts 4(3), 136-143. https://doi. org/10.1037/a0017379 (2010).

11. Mednick, S. A. The associative basis of the creative process. Psychol. Rev. 69, 220-232. https://doi.org/10.1037/h0048850 (1962).

12. Mohr, C., Graves, R. E., Gianotti, L. R., Pizzagalli, D. \& Brugger, P. Loose but normal: A semantic association study. J. Psycholing. Res. 30(5), 475-483. https://doi.org/10.1023/a:1010461429079 (2001).

13. Benedek, M. et al. To create or to recall? Neural mechanisms underlying the generation of creative new ideas. Neuroimage $\mathbf{8 8}$, 125-133. https://doi.org/10.1016/j.neuroimage.2013.11.021 (2014).

14. Zabelina, D. L. \& Ganis, G. Creativity and cognitive control: Behavioral and ERP evidence that divergent thinking, but not real-life creative achievement, relates to better cognitive control. Neuropsychologia 118(Pt A), 20-28. https://doi.org/10.1016/j.neuropsych ologia.2018.02.014 (2018).

15. Acar, S., Chen, X. \& Cayirdag, N. Schizophrenia and creativity: A meta-analytic review. Schizophr. Res. 195, 23-31. https://doi. org/10.1016/j.schres.2017.08.036 (2018).

16. Carson, S. H. Creativity and psychopathology: A shared vulnerability model. Can. J. Psychiatry. 56(3), 144-153. https://doi.org/ 10.1177/070674371105600304 (2011).

17. Dietrich, A. The mythconception of the mad genius. Front. Psychol. 5, 79. https://doi.org/10.3389/fpsyg.2014.00079 (2014).

18. Fink, A. et al. Creativity and schizotypy from the neuroscience perspective. Cogn. Affect. Behav. Neurosci. 14(1), 378-387. https:// doi.org/10.3758/s13415-013-0210-6 (2014).

19. Kyaga, S. et al. Mental illness, suicide and creativity: 40-year prospective total population study. J. Psychiatr. Res. 47(1), 83-90. https://doi.org/10.1016/j.jpsychires.2012.09.010 (2013).

20. Benedek, M., Panzierer, L., Jauk, E. \& Neubauer, A. C. Creativity on tap? Effects of alcohol intoxication on creative cognition. Conscious. Cogn. 56, 128-134. https://doi.org/10.1016/j.concog.2017.06.020 (2017).

21. Hu, S., Ide, J. S., Zhang, S. \& Li, C.-S.R. The right superior frontal gyrus and individual variation in proactive control of impulsive response. J. Neurosci. 36(50), 12688-12696. https://doi.org/10.1523/JNEUROSCI.1175-16.2016 (2016).

22. Beaty, R. E. et al. Creativity and the default network: A functional connectivity analysis of the creative brain at rest. Neuropsychologia 64, 92-98. https://doi.org/10.1016/j.neuropsychologia.2014.09.019 (2014).

23. Flaherty, A. W. Frontotemporal and dopaminergic control of idea generation and creative drive. J. Comp. Neurol. 493(1), 147-153. https://doi.org/10.1002/cne.20768 (2005).

24. Beaty, R. E., Benedek, M., Kaufman, S. B. \& Silvia, P. J. Default and executive network coupling supports creative idea production. Sci. Rep. 5, 10964. https://doi.org/10.1038/srep10964 (2015).

25. Bitsch, F., Berger, P., Nagels, A., Falkenberg, I. \& Straube, B. Impaired right temporoparietal junction-Hippocampus connectivity in schizophrenia and its relevance for generating representations of other minds. Schizophr. Bull. 45(4), 934-945 (2018).

26. Bitsch, F., Berger, P., Nagels, A., Falkenberg, I. \& Straube, B. The role of the right temporo-parietal junction in social decisionmaking. Hum. Brain Mapp. 39(7), 3072-3085 (2018).

27. Buckner, R. L., Andrews-Hanna, J. R. \& Schacter, D. L. The brain's default network: Anatomy, function, and relevance to disease. Ann. N. Y. Acad. Sci. 1124, 1-38. https://doi.org/10.1196/annals.1440.011 (2008).

28. Nusbaum, E. C., Silvia, P. J. \& Beaty, R. E. Ha ha? Assessing individual differences in humor production ability. Psychol. Aesthet. Creat. Arts 11(2), 231-241. https://doi.org/10.1037/aca0000086 (2017).

29. Chan, Y. C. et al. Towards a neural circuit model of verbal humor processing: An fMRI study of the neural substrates of incongruity detection and resolution. Neuroimage 66, 169-176 (2013).

30. Samson, A. C., Hempelmann, C. F., Huber, O. \& Zysset, S. Neural substrates of incongruity-resolution and nonsense humor. Neuropsychologia 47(4), 1023-1033. https://doi.org/10.1016/j.neuropsychologia.2008.10.028 (2009).

31. Vrticka, P., Black, J. M. \& Reiss, A. L. The neural basis of humour processing. Nat. Rev. Neurosci. 14(12), 860-868. https://doi.org/ $10.1038 /$ nrn3566 (2013).

32. Ehrlich, I. et al. Amygdala inhibitory circuits and the control of fear memory. Neuron 62(6), 757-771. https://doi.org/10.1016/j. neuron.2009.05.026 (2009)

33. Iidaka, T. Humor appreciation involves parametric and synchronized activity in the medial prefrontal cortex and hippocampus. Cereb. Cortex (New York) 27(12), 5579-5591. https://doi.org/10.1093/cercor/bhw325 (2017).

34. Berger, P., Bitsch, F., Nagels, A., Straube, B. \& Falkenberg, I. Personality modulates amygdala and insula connectivity during humor appreciation: An event-related fMRI study. Soc. Neurosci. 13(6), 756-768. https://doi.org/10.1080/17470919.2017.1403375 (2018).

35. Babaev, O., Piletti Chatain, C. \& Krueger-Burg, D. Inhibition in the amygdala anxiety circuitry. Exp. Mol. Med. 50(4), 1. https:// doi.org/10.1038/s12276-018-0063-8 (2018).

36. Gold, A. L., Morey, R. A. \& McCarthy, G. Amygdala-prefrontal cortex functional connectivity during threat-induced anxiety and goal distraction. Biol. Psychiatry 77(4), 394-403 (2015).

37. Fink, A., Schwab, D. \& Papousek, I. Sensitivity of EEG upper alpha activity to cognitive and affective creativity interventions. Int. J. Psychophysiol. 82(3), 233-239. https://doi.org/10.1016/j.ijpsycho.2011.09.003 (2011).

38. Shammi, P. \& Stuss, D. T. Humour appreciation: A role of the right frontal lobe. Brain 122(4), 657-666 (1999).

39. Campbell, D. W. et al. The neural basis of humour comprehension and humour appreciation: The roles of the temporoparietal junction and superior frontal gyrus. Neuropsychologia 79, 10-20 (2015).

40. Ochsner, K. N., Silvers, J. A. \& Buhle, J. T. Functional imaging studies of emotion regulation: A synthetic review and evolving model of the cognitive control of emotion. Ann. N. Y. Acad. Sci. 1251, E1-24. https://doi.org/10.1111/j.1749-6632.2012.06751.x (2012). 
41. Martin, R. A. The situational humor response questionnaire (SHRQ) and coping humor scale (CHS): A decade of research findings. Humor 9, 251-272 (1996).

42. Nakamura, T. et al. The role of the amygdala in incongruity resolution: The case of humor comprehension. Soc. Neurosci. 13(5), $553-565$ (2018).

43. Groborz, M. \& Necka, E. Creativity and cognitive control: Explorations of generation and evaluation skills. Creat. Res. J. 15(2-3), 183-197. https://doi.org/10.1080/10400419.2003.9651411 (2003).

44. Green, M. J., Cahill, C. M. \& Malhi, G. S. The cognitive and neurophysiological basis of emotion dysregulation in bipolar disorder. J. Affect. Disord. 103(1-3), 29-42. https://doi.org/10.1016/j.jad.2007.01.024 (2007).

45. Hajek, T., Alda, M., Hajek, E. \& Ivanoff, J. Functional neuroanatomy of response inhibition in bipolar disorders-combined voxel based and cognitive performance meta-analysis. J. Psychiatr. Res. 47(12), 1955-1966. https://doi.org/10.1016/j.jpsychires.2013.08. 015 (2013).

46. Depue, B. E., Burgess, G. C., Willcutt, E. G., Ruzic, L. \& Banich, M. T. Inhibitory control of memory retrieval and motor processing associated with the right lateral prefrontal cortex: Evidence from deficits in individuals with ADHD. Neuropsychologia 48(13), 3909-3917. https://doi.org/10.1016/j.neuropsychologia.2010.09.013 (2010).

47. Morein-Zamir, S. et al. Hypoactivation in right inferior frontal cortex is specifically associated with motor response inhibition in adult ADHD. Hum. Brain Mapp. 35(10), 5141-5152 (2014).

48. Hughes, M. E., Fulham, W. R., Johnston, P. J. \& Michie, P. T. Stop-signal response inhibition in schizophrenia: Behavioural, eventrelated potential and functional neuroimaging data. Biol. Psychol. 89(1), 220-231 (2012).

49. Zandbelt, B. B., van Buuren, M., Kahn, R. S. \& Vink, M. Reduced proactive inhibition in schizophrenia is related to corticostriatal dysfunction and poor working memory. Biol. Psychiatry 70(12), 1151-1158 (2011).

50. Clark, L. \& Sahakian, B. J. Cognitive neuroscience and brain imaging in bipolar disorder. Dial. Clin. Neurosci. 10(2), 153 (2008).

51. Plichta, M. M. et al. Neural hyporesponsiveness and hyperresponsiveness during immediate and delayed reward processing in adult attention-deficit/hyperactivity disorder. Biol. Psychiatry 65(1), 7-14 (2009).

52. Kim, M. J. et al. The structural and functional connectivity of the amygdala: From normal emotion to pathological anxiety. Behav. Brain Res. 223(2), 403-410. https://doi.org/10.1016/j.bbr.2011.04.025 (2011).

53. Townsend, J. D. et al. Frontal-amygdala connectivity alterations during emotion downregulation in bipolar I disorder. Biol. Psychiatry 73(2), 127-135. https://doi.org/10.1016/j.biopsych.2012.06.030 (2013).

54. Li, F. et al. The cognitive up- and down-regulation of positive emotion: Evidence from behavior, electrophysiology, and neuroimaging. Biol. Psychol. 136, 57-66. https://doi.org/10.1016/j.biopsycho.2018.05.013 (2018).

55. LeDoux, J. E. Emotion circuits in the brain. Annu. Rev. Neurosci. 23, 155-184. https://doi.org/10.1146/annurev.neuro.23.1.155 (2000).

56. Quirk, G. J. \& Gehlert, D. R. Inhibition of the amygdala: Key to pathological states?. Ann. N. Y. Acad. Sci. 985, 263-272. https:// doi.org/10.1111/j.1749-6632.2003.tb07087.x (2003).

57. Yonelinas, A. P. \& Ritchey, M. The slow forgetting of emotional episodic memories: An emotional binding account. Trends Cogn. Sci. 19(5), 259-267. https://doi.org/10.1016/j.tics.2015.02.009 (2015).

58. Elliott, M. L. et al. What is the test-retest reliability of common task-fMRI measures? New empirical evidence and a meta-analysis. Psychol. Sci. 31, 792 (2020).

59. Dubois, J. \& Adolphs, R. Building a science of individual differences from fMRI. Trends Cogn. Sci. 20(6), 425-443 (2016).

60. Friston, K. Ten ironic rules for non-statistical reviewers. Neuroimage 61(4), 1300-1310 (2012).

61. Oldfield, R. C. The assessment and analysis of handedness: The Edinburgh inventory. Neuropsychologia 9(1), 97-113 (1971).

62. Schmidt, T., \& Schütte, W. Folker: An Annotation Tool for Efficient Transcription of Natural, Multi-party Interaction (2010).

63. Stroop, J. R. Studies of interference in serial verbal reactions. J. Exp. Psychol. 18(6), 643 (1935).

64. Gross, J. J. \& John, O. P. Individual differences in two emotion regulation processes: Implications for affect, relationships, and well-being. J. Pers. Soc. Psychol. 85(2), 348-362. https://doi.org/10.1037/0022-3514.85.2.348 (2003).

65. Slotnick, S. D., Schwarzbach, J. \& Yantis, S. Attentional inhibition of visual processing in human striate and extrastriate cortex. Neuroimage 19(4), 1602-1611 (2003).

66. McLaren, D. G., Ries, M. L., Xu, G. \& Johnson, S. C. A generalized form of context-dependent psychophysiological interactions (gPPI): A comparison to standard approaches. Neuroimage 61(4), 1277-1286 (2012).

67. Pernet, C. R., Wilcox, R. R. \& Rousselet, G. A. Robust correlation analyses: False positive and power validation using a new open source matlab toolbox. Front. Psychol. 3, 606 (2013).

68. Wilcox, R. Inferences based on a skipped correlation coefficient. J. Appl. Stat. 31(2), 131-143 (2004).

\section{Acknowledgements}

This work was supported by the Core Facility Brain Imaging, Faculty of Medicine, University of Marburg, Else Kröner-Fresenius-Stiftung (Grant Number: 2014_A136) and Deutsche Forschungsgemeinschaft (Grant Numbers: STR-1146/4-1, STR-1146/8-1, STR-1146/9-1, to BS).

\section{Author contributions}

F.B.: Designed the study, collected the data, conducted the data analyses, programmed the stroop task and wrote the first version of the manuscript. P.B.: Designed the study, collected the data, edited further versions of the manuscript. A.F.: Designed the study, designed the adapted AU task, edited further versions of the manuscript. A.N. and B.S.: Designed the study, raised the project funding and supervised the entire project, edited further versions of the manuscript. I.F.: Designed the study, raised the project funding and supervised the entire project, edited further versions of the manuscript. All authors have approved the submitted version of the manuscript.

\section{Funding}

Open Access funding enabled and organized by Projekt DEAL.

\section{Competing interests}

The authors declare no competing interests.

\section{Additional information}

Supplementary Information The online version contains supplementary material available at https://doi.org/ 10.1038/s41598-021-89843-8.

Correspondence and requests for materials should be addressed to F.B. 
Reprints and permissions information is available at www.nature.com/reprints.

Publisher's note Springer Nature remains neutral with regard to jurisdictional claims in published maps and institutional affiliations.

(c) (i) Open Access This article is licensed under a Creative Commons Attribution 4.0 International License, which permits use, sharing, adaptation, distribution and reproduction in any medium or format, as long as you give appropriate credit to the original author(s) and the source, provide a link to the Creative Commons licence, and indicate if changes were made. The images or other third party material in this article are included in the article's Creative Commons licence, unless indicated otherwise in a credit line to the material. If material is not included in the article's Creative Commons licence and your intended use is not permitted by statutory regulation or exceeds the permitted use, you will need to obtain permission directly from the copyright holder. To view a copy of this licence, visit http://creativecommons.org/licenses/by/4.0/.

(C) The Author(s) 2021, corrected publication 2021 